\title{
Asian-Pacific perspective on the psychological well-being of healthcare workers during the evolution of the COVID-19 pandemic
}

Nicholas W. S. Chew*, Jinghao Nicholas Ngiam*, Benjamin Yong-Qiang Tan*, Sai-Meng Tham, Celine Yan-Shan Tan, Mingxue Jing, Renarebecca Sagayanathan, Jin Tao Chen, Lily Y. H. Wong, Aftab Ahmad, Faheem Ahmed Khan, Maznah Marmin, Fadhlina Binte Hassan, Tai Mei-Ling Sharon, Chin Han Lim, Mohamad Iqbal Bin Mohaini, Rivan Danuaji, Thang H. Nguyen, Georgios Tsivgoulis, Sotirios Tsiodras, Paraskevi C. Fragkou, Dimitra Dimopoulou, Arvind K. Sharma, Kenam Shah, Bhargesh Patel, Suktara Sharma, R. N. Komalkumar, R. V. Meenakshi, Shikha Talati, Hock Luen Teoh, Cyrus S. Ho, Roger C. Ho and Vijay K. Sharma

\section{Background}

The coronavirus disease 2019 (COVID-19) pandemic has led to significant strain on front-line healthcare workers.

\section{Aims}

In this multicentre study, we compared the psychological outcomes during the COVID-19 pandemic in various countries in the Asia-Pacific region and identified factors associated with adverse psychological outcomes.

\section{Method}

From 29 April to 4 June 2020, the study recruited healthcare workers from major healthcare institutions in five countries in the Asia-Pacific region. A self-administrated survey that collected information on prior medical conditions, presence of symptoms, and scores on the Depression Anxiety Stress Scales and the Impact of Events Scale-Revised were used. The prevalence of depression, anxiety, stress and post-traumatic stress disorder (PTSD) relating to COVID-19 was compared, and multivariable logistic regression identified independent factors associated with adverse psychological outcomes within each country.

\section{Results}

A total of 1146 participants from India, Indonesia, Singapore, Malaysia and Vietnam were studied. Despite having the lowest volume of cases, Vietnam displayed the highest prevalence of
PTSD. In contrast, Singapore reported the highest case volume, but had a lower prevalence of depression and anxiety. In the multivariable analysis, we found that non-medically trained personnel, the presence of physical symptoms and presence of prior medical conditions were independent predictors across the participating countries

\section{Conclusions}

This study highlights that the varied prevalence of psychological adversity among healthcare workers is independent of the burden of COVID-19 cases within each country. Early psychological interventions may be beneficial for the vulnerable groups of healthcare workers with presence of physical symptoms, prior medical conditions and those who are not medically trained.

\section{Keywords}

COVID-19; healthcare workers; psychological impact; pandemic; Asia-Pacific.

\section{Copyright and usage}

(c) The Author(s), 2020. Published by Cambridge University Press on behalf of the Royal College of Psychiatrists. This is an Open Access article, distributed under the terms of the Creative Commons Attribution licence (http://creativecommons.org/ licenses/by/4.0/), which permits unrestricted re-use, distribution, and reproduction in any medium, provided the original work is properly cited
After the initial presentation of a cluster of viral pneumonia in Wuhan, China, coronavirus disease 2019 (COVID-19) has spread rapidly and globally, with the outbreak at its peak in various countries in South Asia. ${ }^{1}$ Accordingly, as of the 30th June 2020, Singapore reported a total of 4846 cases per million population with 4 deaths per million population. This was followed by Malaysia (255 cases and 4 deaths per million population ); India (164 cases and 5 deaths per million population); Indonesia (105 cases and 5 deaths per million population ) and Vietnam (3 cases per million population and no reported deaths). ${ }^{2}$

Besides the direct health impact of COVID-19 on patients, ${ }^{3}$ it has placed a significant strain on healthcare workers and resources. Healthcare institutions have enforced measures such as the donning of appropriate personal protective equipment and minimising nonessential services to avoid unnecessary exposure of healthcare staff to COVID-19. ${ }^{4}$ It has led to considerable psychological impact on front-line healthcare staff even during the early periods of the outbreak. $^{5-9}$ In this multicentre study on healthcare workers, we

* Joint first authors. compared the psychological outcomes and its predictors during the current COVID-19 pandemic in various countries in the AsiaPacific region.

\section{Method}

\section{Study setting and population}

Between the period of 29 April 2020 to 4 June 2020, healthcare workers from major tertiary healthcare institutions from India, Singapore, Malaysia, Vietnam and Indonesia were recruited. During the study period, these healthcare institutions were actively involved in the management of COVID-19 patients. The survey was extended to all healthcare workers directly involved in wards or facilities designated for managing patients with COVID-19. Study participants included medically trained (doctors and nurses) and non-medically trained personnel (administrative staff, pharmacists, cleaners, porters and technicians). All participants provided written informed consent. The survey was administered once and there were no subsequent follow-up questionnaires. The study was 
approved by the institutional review boards of various centres, in accordance with the principles of the Declaration of Helsinki.

\section{Screening questionnaire}

The study questionnaire was administered in English. In all the hospitals where the survey was administered, English was the primary language of communication among the staff. The participants' baseline information on demographic characteristics, their past medical history and symptom prevalence in the immediate previous month were obtained. Psychological outcomes were assessed using Depression Anxiety Stress Scales (DASS-21) and the Impact of Events Scale-Revised (IES-R) instruments. ${ }^{10,11}$

The DASS-21 is an internationally recognised screening tool used for the general population for screening depression, anxiety and stress. It is a self-administered 21 -item instrument created by the University of New South Wales, Australia, which screens for depression, anxiety and stress based on the recommended severity thresholds for the depression, anxiety, stress subscales. ${ }^{10}$ For the purpose of this study, we examined depression, anxiety and stress with cut-off scores of $>9,>7$ and $>14$, respectively. Each DASS-21 subscales were further categorised as mild, moderate, severe and extremely severe, as follows: for depression subscales, 10-13, 1420, 21-27, 28-42 points, respectively; for anxiety subscales, $8-9$, $10-14,15-19,20-42$ points, respectively; and for stress subscales, $15-18,19-25,26-33,34-42$ points, respectively. ${ }^{10}$

The IES- $\mathrm{R}$ was used to assess the extent of psychological distress among healthcare workers. This is an internationally validated 22item screening system that has three further subcategories (intrusion, avoidance, and hyperarousal), which are associated with the symptoms displayed in post-traumatic stress disorder (PTSD). ${ }^{11}$ We studied PTSD specifically in relation to the COVID-19 pandemic, and did not examine other general causes of PTSD. Participants had to rate their level of distress for each component during the past 7 days on the questionnaire. The severity of psychological impact was graded from normal (0-23 points), mild (24-32 points), moderate (33-36 points), to severe ( $>37$ points). A threshold score of $\geq 24$ points was used to define PTSD as a clinical concern. ${ }^{11}$ Both DASS-21 and IES-R have been used to evaluate the psychological impact of COVID-19 in previous studies. ${ }^{5,12}$

\section{Study outcomes}

The primary study outcome was the comparison of the prevalence of depression, anxiety, stress and PTSD related to COVID-19 reported by healthcare workers during the pandemic in five countries in the Asia-Pacific region. Subsequently, we explored the independent predictors of these psychological outcomes in each country.

\section{Statistical analyses}

Continuous variables were expressed as mean and s.d., while categorical variables were expressed as absolute values (percentage). Continuous variables were compared using Student's $t$-test, while categorical variables were examined using Pearson's chi-squared test (or Fisher's exact test, where appropriate). The comparison of the prevalence of psychological outcomes among the five countries was performed using logistic regression. The stress outcome was excluded from the logistic regression model as none of the healthcare workers in Singapore, India and Malaysia were screened positive for the outcome. Multivariable logistic regression was performed for the subgroup analysis of each country to evaluate for independent associations with adverse psychological outcome and traditional covariates. A $P$-value of less than 0.05 was deemed significant for this study. All statistical analyses were performed using IBM SPSS Statistics for Windows, Version 25.0.
Results

\section{Participants}

Of the 1300 healthcare workers invited to participate in this study, 1146 ( $88.2 \%$ response rates) agreed to participate in the study. There were $384(33.5 \%)$ respondents from India, 277 (24.2\%) from Singapore, 250 (21.8\%) from Indonesia, 175 (15.3) from Malaysia and $60(5.2 \%)$ from Vietnam. The majority of participants were female $(65.1 \%)$ and married $(54.2 \%)$, with a mean age of 31.7 (s.d. =7.8) years. Notably, the study cohort from India was younger $(27.7$ years, s.d. $=5.7)$ compared with the general study population. The majority of participants were medically trained (755, 65.8\%), of which $58.8 \%$ (444) were nurses and the remaining $41.2 \%$ (311) physicians. Of the non-medically trained personnel $(n=391)$, most were clerical staff $(167,14.6 \%)$ (Table 1$)$.

The commonest reported symptoms were lethargy $(415,36.2 \%)$ and headache (384, 33.5\%) (Fig. 1). Almost one-third (375, 32.7\%) of participants did not have any symptoms in the 1 month prior to survey administration. On the other hand, $124(10.8 \%)$ reported one symptom, 119 (10.4\%) reported two symptoms and 528 (46.1\%) reported three or more symptoms. Most of the reported symptoms were rated as mild in severity.

\section{Comparison of adverse psychological outcomes between countries}

During each of the countries' recruitment period, the total confirmed cases per day per 1 million population and deaths per day/ per 1 million population were collated (Figs. 2 and 3). Singapore had the highest number of cases (109.6 per day/1 million population) followed by Malaysia (1.9 cases per day/1 million population), Indonesia ( 1.8 cases per day/ 1 million population), India ( 1.2 cases per day/1 million population) and Vietnam ( 0.03 cases per day/1 million population).

During the COVID-19 pandemic in various Asia-Pacific countries, 51 (4.5\%) of participants of our overall study cohort of healthcare workers screened positive for depression, 60 (5.2\%) for anxiety, 12 (1.0\%) for stress, and 91 (7.9\%) for PTSD related to COVID- 19. The overall mean DASS-21 depression, anxiety and stress scores were 1.89 (s.d. = 3.10), 1.95 (s.d.=2.75), and 2.75 (s.d. =3.50), respectively. The mean total IES-R score was 8.92 (s.d.=9.76), while the mean scores for IES-R subsets for intrusion, avoidance and hyperarousal were 0.42 (s.d. $=0.47), 0.42$ (s.d. =0.52) and 0.40 (s.d. $=0.47$ ), respectively.

In the individual country subgroup analysis, India displayed the lowest prevalence of adverse psychological outcomes. India had the lowest prevalence of depression $(0.8 \%)$, followed by Indonesia (2.4\%) Singapore (4.7\%), Vietnam (6.7\%) and Malaysia (14.3\%). Again, India had the lowest prevalence $(0.8 \%)$ of anxiety, followed by Singapore (3.6\%), Vietnam (6.7\%) Indonesia (6.8\%) and Malaysia (14.9\%). Interestingly, the prevalence of stress was low in most countries, with figures of $5.7 \%$ in Indonesia and 3.3\% in Vietnam, but none screened positive in Singapore and India (Fig. 4). Overall, there were significant differences in the prevalence of adverse psychological outcomes between the five countries. Using India as the reference group, overall results are displayed in Table 2.

The IES-R system was used to evaluate the extent of psychological distress among healthcare workers. Healthcare workers from India displayed the lowest prevalence of PTSD related to COVID-19 (2.1\%), followed by Malaysia (6.3\%), Indonesia (11.6\%), Singapore (12.3\%) and Vietnam (15.0\%). The comparison of IES-R subsets of intrusion, avoidance and hyperarousal between the five countries are displayed in Fig. 5. 


\begin{tabular}{|c|c|c|c|c|c|c|}
\hline Characteristics & Overall $(n=1146)$ & Singapore $(n=277)$ & India $(n=384)$ & Malaysia $(n=175)$ & Vietnam $(n=60)$ & Indonesia $(n=250)$ \\
\hline Female gender, $n$ (\%) & $746(65.1)$ & $193(69.7)$ & $251(65.4)$ & $118(67.4)$ & $44(73.3)$ & $140(56.0)$ \\
\hline Age, mean (s.d.) & $31.7(7.8)$ & $35.0(9.2)$ & $27.7(5.7)$ & $32.4(6.5)$ & $34.7(9.6)$ & $33.2(7.0)$ \\
\hline \multicolumn{7}{|l|}{ Ethnicity, $n(\%)$} \\
\hline Chinese & $154(13.4)$ & $136(49.1)$ & $0(0.0)$ & $12(6.9)$ & $0(0.0)$ & $6(2.4)$ \\
\hline Indian & 435 (38.0) & $46(16.6)$ & $383(99.7)$ & $6(3.4)$ & $0(0.0)$ & $0(0.0)$ \\
\hline Malay & $211(18.4)$ & $29(10.5)$ & $1(0.3)$ & 58 (33.1) & $0(0.0)$ & $123(49.2)$ \\
\hline Eurasian & $3(0.3)$ & $2(0.7)$ & $0(0.0)$ & $0(0.0)$ & $0(0.0)$ & $1(0.4)$ \\
\hline White & $28(2.4)$ & $25(9.0)$ & $0(0.0)$ & $0(0.0)$ & $3(5.0)$ & $0(0.0)$ \\
\hline Other & 315 (27.5) & $39(14.1)$ & $0(0.0)$ & $99(56.6)$ & $57(95.0)$ & $120(48.0)$ \\
\hline \multicolumn{7}{|l|}{ Marital status } \\
\hline Single & $504(44.0)$ & $122(44.0)$ & $237(61.7)$ & $66(37.7)$ & $24(40.0)$ & $55(22.0)$ \\
\hline Married & $621(54.2)$ & $145(52.4)$ & $146(38.0)$ & $103(58.9)$ & $35(58.3)$ & $192(76.8)$ \\
\hline Divorced/separated/widowed & $21(1.8)$ & $10(3.6)$ & $1(0.3)$ & $6(3.4)$ & $1(1.7)$ & $3(1.2)$ \\
\hline \multicolumn{7}{|l|}{ Occupation } \\
\hline Physician & $311(27.1)$ & $77(27.8)$ & $84(21.9)$ & $62(35.4)$ & $28(46.7)$ & $60(24)$ \\
\hline Nurse & $444(38.7)$ & $99(35.7)$ & $167(43.5)$ & $94(53.7)$ & $20(33.3)$ & $64(25.6)$ \\
\hline Technician & $70(6.1)$ & $21(7.6)$ & $42(10.9)$ & $3(1.7)$ & $4(6.7)$ & $0(0.0)$ \\
\hline Clerical staff/executive & $167(14.6)$ & $14(5.1)$ & $40(10.4)$ & $3(1.7)$ & $5(8.3)$ & $105(42.0)$ \\
\hline Administrator & $48(4.2)$ & $16(5.8)$ & $24(6.3)$ & $6(3.4)$ & $2(3.3)$ & $0(0.0)$ \\
\hline Maintenance worker & $33(2.9)$ & $9(3.2)$ & $21(5.5)$ & $2(1.1)$ & $1(1.7)$ & $0(0.0)$ \\
\hline Allied professional & $73(6.4)$ & $41(14.8)$ & $6(1.6)$ & $5(2.9)$ & $0(0.0)$ & $21(8.4)$ \\
\hline \multicolumn{7}{|l|}{ Prior medical conditions } \\
\hline Hypertension & $65(5.7)$ & $15(5.4)$ & $10(2.6)$ & $19(10.9)$ & $5(8.3)$ & $16(6.4)$ \\
\hline Dyslipidaemia & $43(3.8)$ & $7(2.5)$ & $6(1.6)$ & $7(4.0)$ & $5(8.3)$ & $18(7.2)$ \\
\hline Diabetes mellitus & $23(2.0)$ & $6(2.2)$ & $3(0.8)$ & $4(2.3)$ & $5(8.3)$ & $5(2.0)$ \\
\hline Asthma & $60(5.2)$ & $15(5.4)$ & $6(1.6)$ & $13(7.4)$ & $1(1.7)$ & $25(10)$ \\
\hline Eczema & $43(3.8)$ & $16(5.8)$ & $0(0)$ & $5(2.9)$ & $3(5)$ & $19(7.6)$ \\
\hline Migraine & $109(9.5)$ & $26(9.4)$ & $20(5.2)$ & $17(9.7)$ & $5(8.3)$ & $41(16.4)$ \\
\hline Ischaemic heart disease & $6(0.5)$ & $0(0.0)$ & $1(0.3)$ & $0(0.0)$ & $0(0.0)$ & $5(2.0)$ \\
\hline Stroke & $3(0.3)$ & $0(0.0)$ & $0(0.0)$ & $1(0.6)$ & $0(0.0)$ & $2(0.8)$ \\
\hline Others & $32(2.8)$ & $16(5.8)$ & $2(0.5)$ & $0(0.0)$ & $3(5)$ & $11(4.4)$ \\
\hline Smoking & 37 (3.2) & $17(6.1)$ & $11(2.9)$ & $2(1.1)$ & $1(1.7)$ & $6(2.4)$ \\
\hline \multicolumn{7}{|l|}{ Physical symptoms } \\
\hline Throat pain & $289(25.2)$ & $58(20.9)$ & $143(37.2)$ & $26(14.9)$ & $10(16.7)$ & $52(20.8)$ \\
\hline Nausea & $88(7.7)$ & $11(4.0)$ & $40(10.4)$ & $7(4.0)$ & 0 & $30(12.0)$ \\
\hline Anxiety & $367(32.0)$ & $80(28.9)$ & $121(31.5)$ & $33(18.9)$ & $28(46.7)$ & $105(42.0)$ \\
\hline Insomnia & $302(26.4)$ & $82(29.6)$ & $106(27.6)$ & $43(24.6)$ & $22(36.7)$ & $49(19.6)$ \\
\hline Poor appetite & $218(19.0)$ & $25(9.0)$ & $122(31.8)$ & $20(11.4)$ & $12(20.0)$ & $39(15.6)$ \\
\hline Lethargy & $415(36.2)$ & $96(34.7)$ & $132(34.4)$ & $50(28.6)$ & $11(18.3)$ & $126(50.4)$ \\
\hline Watery eyes & $48(4.2)$ & $16(5.8)$ & $8(2.1)$ & $8(4.6)$ & $3(5.0)$ & $13(5.2)$ \\
\hline Pruritus & $99(8.6)$ & $23(8.3)$ & $15(3.9)$ & $10(5.7)$ & $5(8.3)$ & $46(18.4)$ \\
\hline Myalgia & $328(28.6)$ & $74(26.7)$ & $136(35.4)$ & $43(24.6)$ & $8(13.3)$ & $67(26.8)$ \\
\hline Rash & $66(5.8)$ & $15(5.4)$ & $20(5.2)$ & $10(5.7)$ & $1(1.7)$ & $20(8.0)$ \\
\hline Coryza & $137(12.0)$ & $74(26.7)$ & 136 (35.4) & $43(24.6)$ & $8(13.3)$ & $67(26.8)$ \\
\hline Breathlessness & $83(7.2)$ & $23(8.3)$ & $31(8.1)$ & $11(6.3)$ & $1(1.7)$ & $17(6.8)$ \\
\hline Cough & $166(14.5)$ & $52(18.8)$ & $10(2.6)$ & $31(17.7)$ & $7(11.7)$ & $66(26.4)$ \\
\hline Sputum & $109(9.5)$ & $38(13.7)$ & $13(3.4)$ & $12(6.9)$ & $7(11.7)$ & $39(15.6)$ \\
\hline Headache & 384 (33.5) & $96(34.7)$ & $158(41.1)$ & $50(28.6)$ & $18(30.0)$ & $62(24.8)$ \\
\hline Neck stiffness & $157(13.7)$ & $53(19.1)$ & $18(4.7)$ & $29(16.6)$ & $6(10.0)$ & $51(20.4)$ \\
\hline
\end{tabular}

\section{Predictors of adverse psychological outcomes}

Multivariate analysis of healthcare workers was performed to assess independent predictors of anxiety for each country, and the variables of the model included gender (female), non-medically trained personnel, presence of prior medical conditions (past medical history), presence of symptoms and age. The multivariate analysis model for depression showed the presence of physical symptoms as the independent predictor of depression in the Malaysian (odds ratio $(\mathrm{OR})=5.673,95 \%$ CI $1.780-18.075, P=$ 0.003 ) and Indonesian cohort $(\mathrm{OR}=1.241,95 \%$ CI 1.010-1.526, $P=0.040)$ (Table 3).

The study found that being a non-medically trained healthcare personnel was an independent predictor for anxiety in the Indonesian cohort $(\mathrm{OR}=4.908,95 \% \mathrm{CI} 1.282-18.789, P=0.020)$ after adjusting for confounders. In the Singapore cohort, the presence of prior medical conditions was an independent predictor for anxiety $(\mathrm{OR}=5.828,95 \% \mathrm{CI} 1.397-24.308, P=0.016)$ (Table 4).
In the multivariate analysis, the independent predictors of PTSD related to COVID-19 were non-medically trained personnel in the Singaporean $(\mathrm{OR}=2.729,95 \% \mathrm{CI} 1.150-6.472, P=0.023)$ and Indonesian cohort $(\mathrm{OR}=2.443,95 \% \mathrm{CI} 1.004-5.942, P=0.049)$. Additionally, the presence of prior medical conditions $(\mathrm{OR}=$ $2.425,95 \%$ CI 1.014-5.802, $P=0.046)$ and presence of symptoms $(\mathrm{OR}=6.692,95 \%$ CI $1.517-29.521, P=0.012)$ were independent predictors of PTSD in the Singaporean cohort (Table 5).

\section{Discussion}

\section{Main findings}

To our knowledge, this is the first multicentre study that has examined the prevalence of psychological outcomes among healthcare workers during the evolution of the COVID-19 pandemic in the Asia-Pacific region. Our study demonstrated the discrepancy 


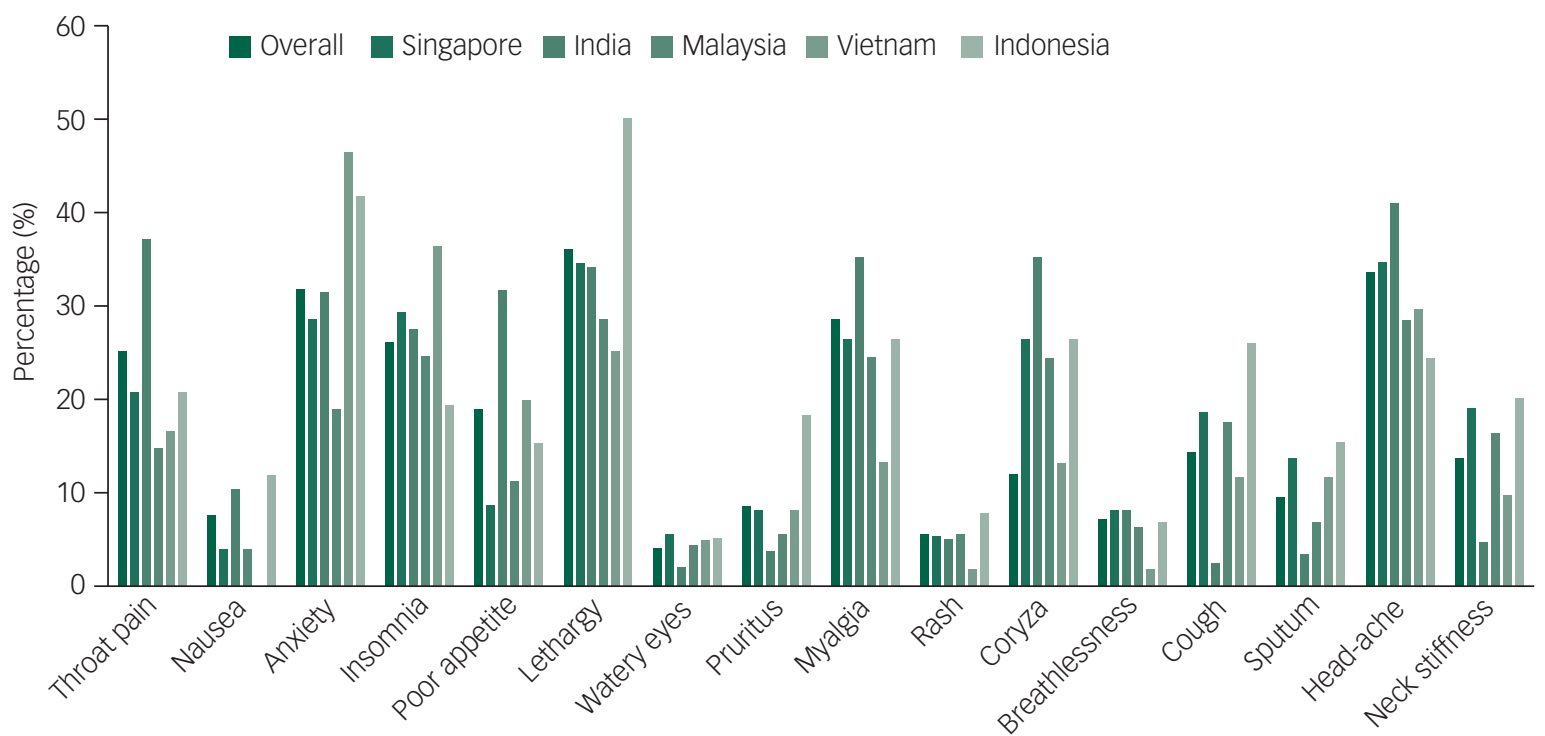

Fig. 1 Prevalence of physical symptoms experienced by healthcare workers by country during the COVID-19 pandemic.

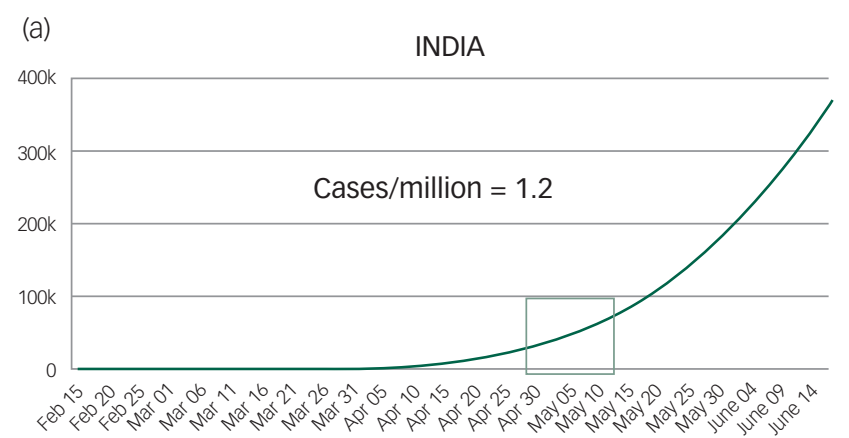

(c)

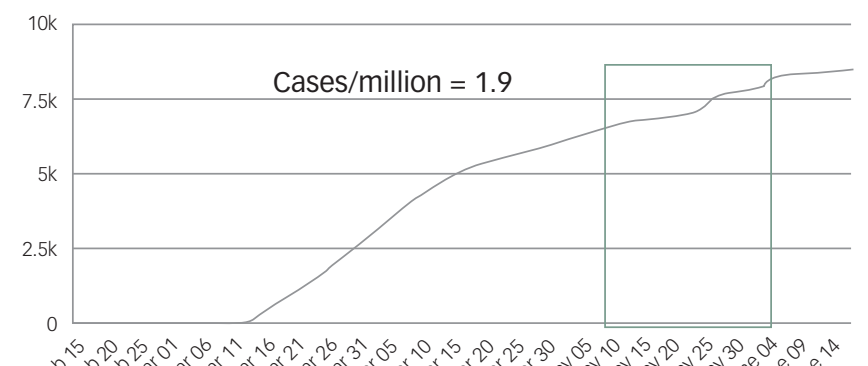

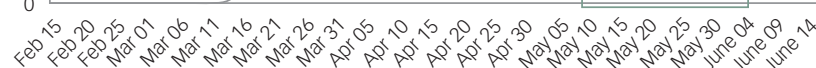

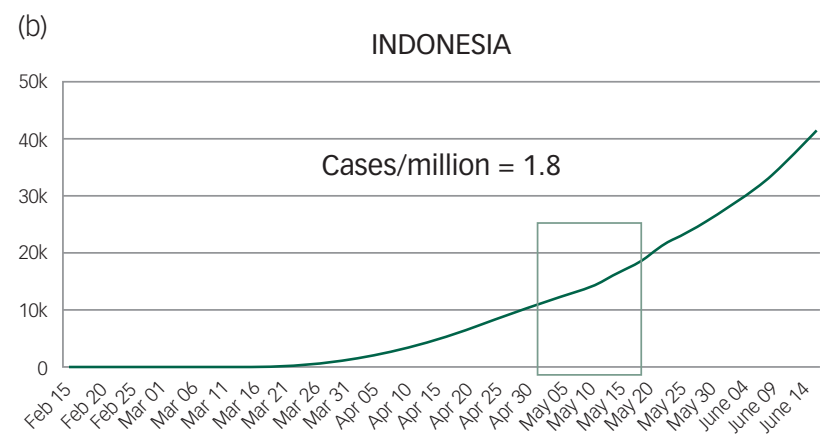

(d)

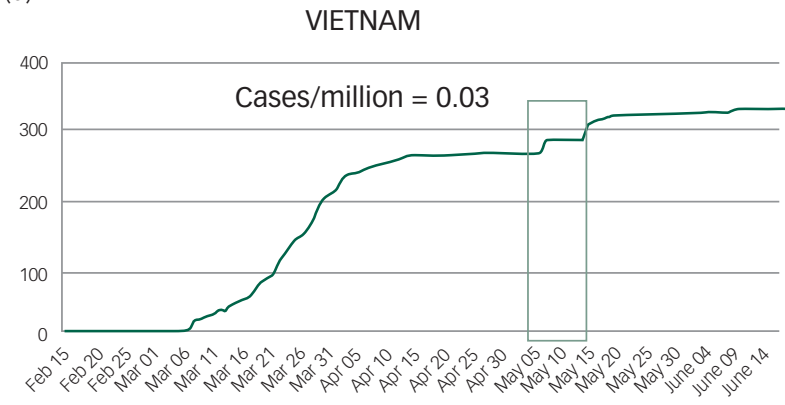

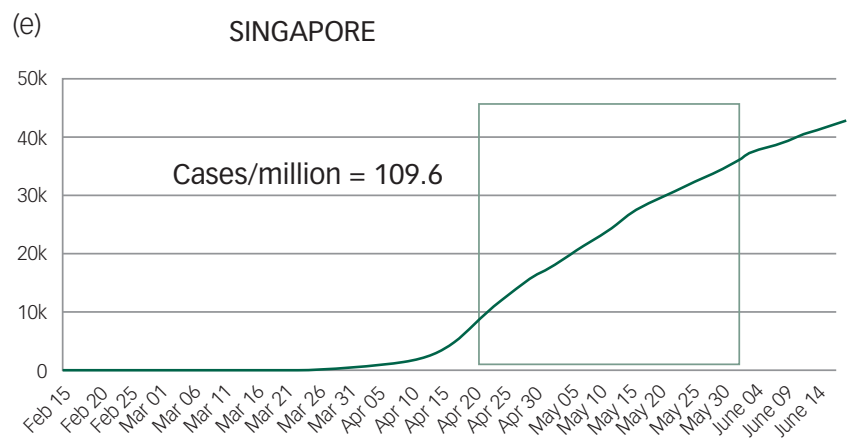

Fig. 2 The longitudinal trajectory of confirmed cases of COVID-19 in the participating countries. Total number of confirmed coVID-19 cases per 1 million population for each country is also represented. 

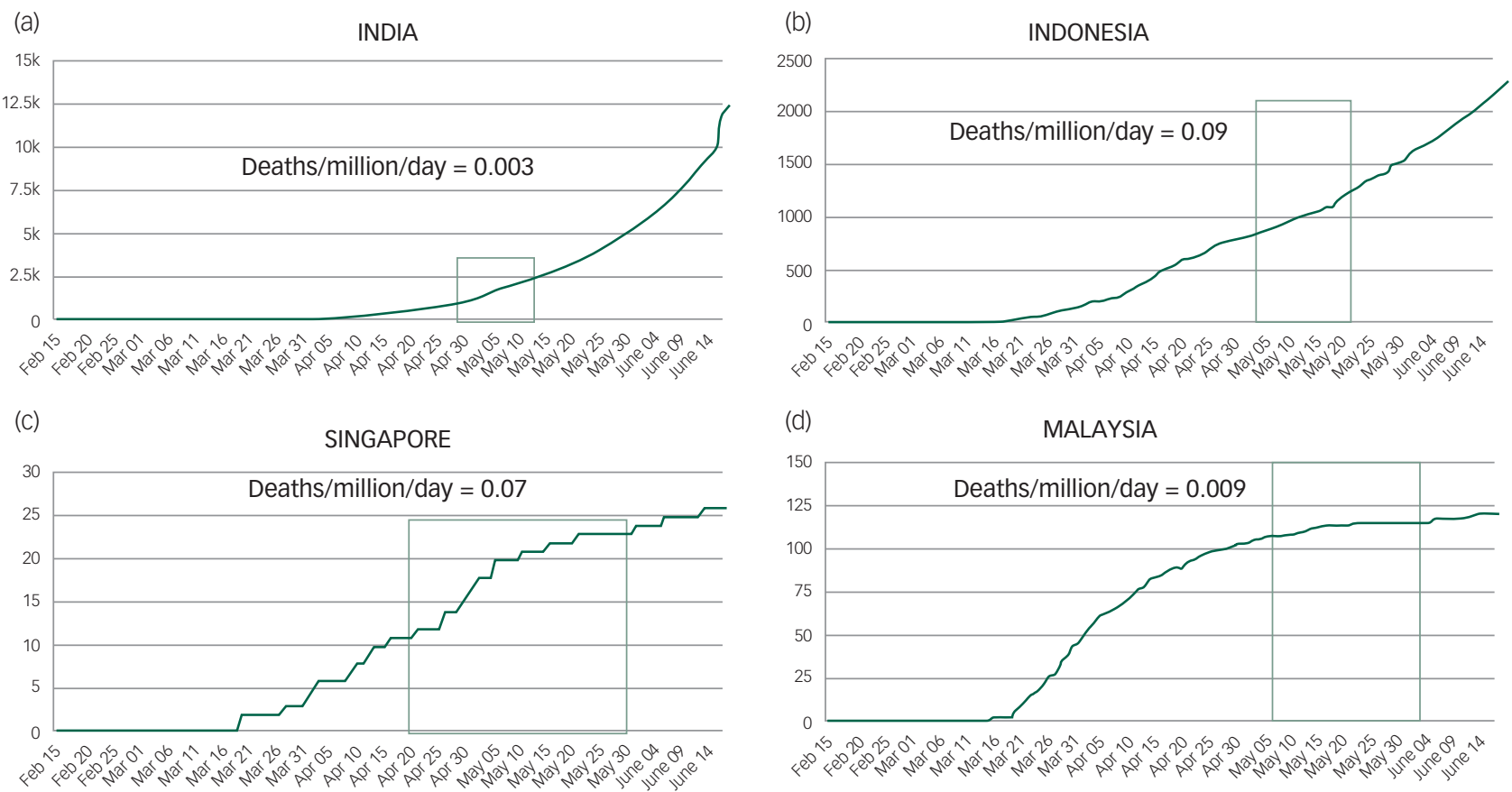

Fig. 3 The longitudinal trajectory of confirmed deaths because of COVID-19 in the participating countries. Total number of confirmed deaths because of COVID-19 per 1 million population for each country is also represented.

(a) India; (b) Indonesia; (c) Singapore; (d) Malaysia. The denoted section of the trend line (green box) signifies the study period of each country.

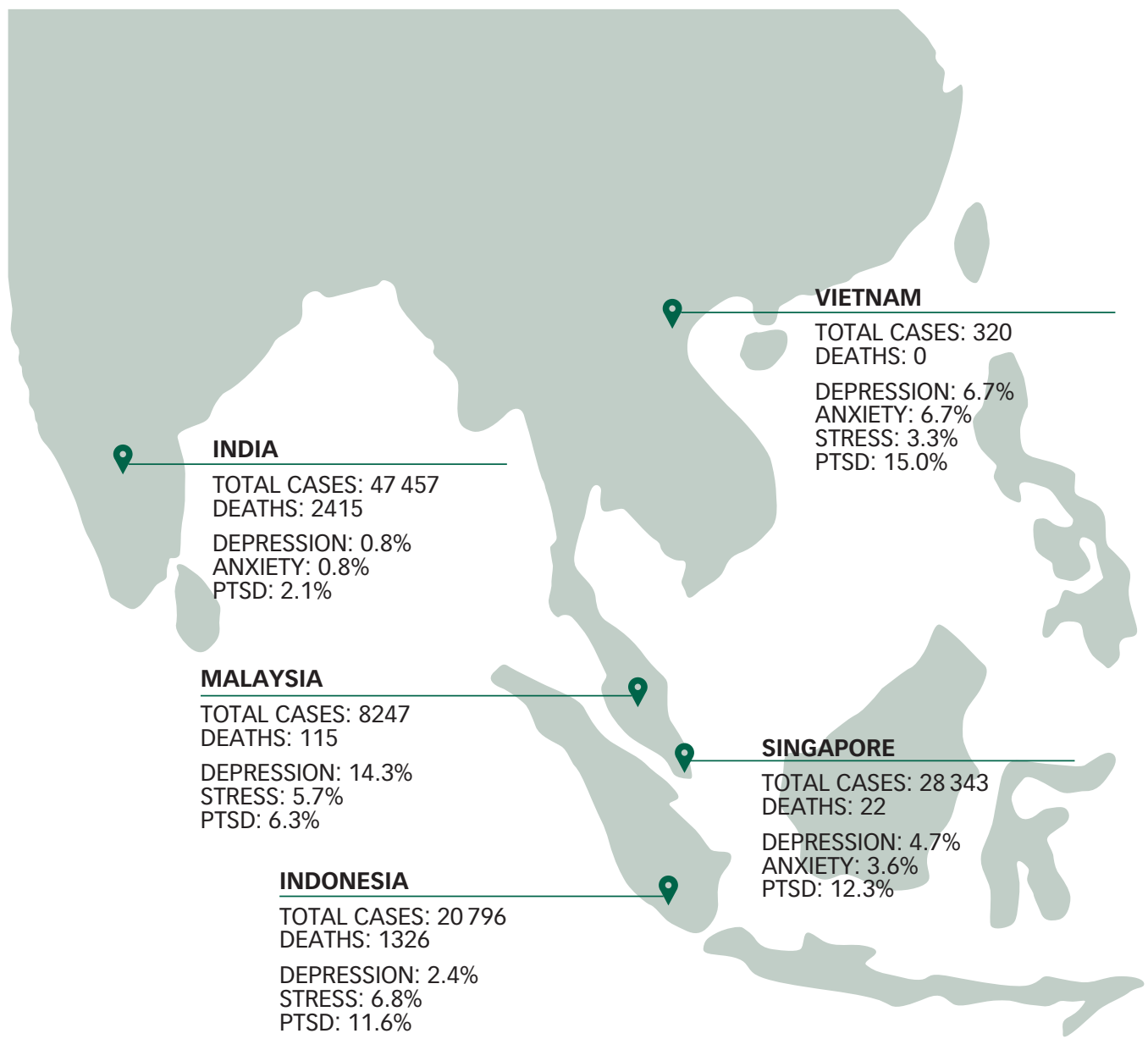

Fig. 4 The total number of confirmed COVID-19 cases and deaths at the end of the country's study period, with the prevalence of adverse psychological outcomes among healthcare workers in each country $(n=1146)$.

The Depression Anxiety Stress Scales (DASS-21) was used, in which DASS-21 cut-off scores of $>9,>7$ and $>14$ indicate a positive screen of depression, anxiety and stress, respectively. Post-traumatic stress disorder was screened using the Impact of Events Scale-Revised (IES-R) score, where a total IES-R cut-off score of 24 is used to classify post-traumatic stress disorder as a clinical concern. India and singapore did not have any healthcare workers screen positive for stress. PTSD, post-traumatic stress disorder. 


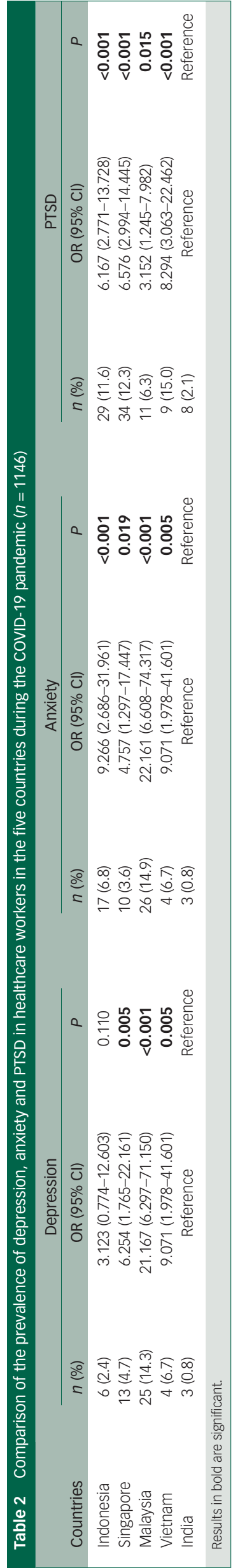

between the volume of confirmed COVID-19 cases per day/1 million population and the prevalence of adverse psychological outcomes in the participating countries. Despite having the lowest volume of cases per day/1 million population, Vietnam displayed a higher prevalence of PTSD related to COVID-19 among healthcare workers compared with India. In contrast, Singapore reported the highest number of cases per day/1 million population, but had a lower prevalence of depression and anxiety among its healthcare workers, when compared with the Malaysian cohort. Our study highlights that all healthcare workers were vulnerable to psychological adversity regardless of the volume of confirmed COVID-19 cases. In fact, it would be crucial to focus efforts on addressing the independent predictors of psychological adversity, such as healthcare workers with the presence of physical symptoms, presence of prior medical conditions and those who are not medically trained.

\section{Comparison with findings from other studies}

During the early stages of the COVID-19 pandemic, Wang et al investigated the psychological impact of the COVID-19 pandemic on the general public in China using the DASS-21 scale, and found that $16.5 \%, 28.8 \%$ and $8.1 \%$ of its respondents reported moderate-severe depressive, anxiety and stress levels, respectively. ${ }^{12}$ However, given the differences in sampling approach, measurement and methodology, it poses a challenge for direct comparison with our present study cohorts' prevalence of depression (1.5\%), anxiety (3.0\%) and stress $(0.09 \%)$. We speculate that the prevalence of adverse psychological outcomes may improve across the COVID19 pandemic trajectory, as there will have been an additional amount of time and effort in countries in building their readiness through resource allocation, emergency implementation of drastic infection control measures and mental preparedness. ${ }^{13,14}$

Our earlier study reported depression rates among non-medical and medical healthcare personnel to be $10.3 \%$ and $8.1 \%$ respectively, as compared with the overall $4.7 \%$ depression rate displayed in the present study. ${ }^{5}$ This trend was similar for the rates of anxiety in the early phases of the pandemic (20.7\% in non-medical and $10.8 \%$ in medical healthcare workers, versus the overall $3.6 \%$ in the present study). ${ }^{5}$ It is important to note that it is difficult to make accurate longitudinal conclusions when comparing the two studies because of the different population cohorts. Nevertheless, there is a trend towards better psychological preparedness and resilience for impending widespread transmission when the spread of the pandemic is already known, as compared with the uncertainty during the earlier stages of the pandemic. ${ }^{15}$

\section{Impact of differing strategies in different countries}

Each of the participating countries has adopted strategies to curb the spread of COVID-19 and ensuring confidence in its healthcare workers through preventive strategies. The world witnessed the largest COVID-19 national lockdown in India, which led to the desired effect of flattening the epidemic curve. India's population of 1.3 billion with its widening socioeconomic disparities pose unique challenges in the pandemic. Moreover, India's young general population (65\% of the population are less than 35 years of age) may serve as a determining factor for psychological resilience as evident by the lower levels of adverse psychological outcomes in this study. ${ }^{16}$ This characteristic is reflected in the present study's Indian cohort with a lower mean age with fewer prior medical conditions as compared with their counterparts.

The most effective lockdown was seen in Vietnam. The country tightened its border immediately after the first death in Wuhan. Vietnam's approach was never based on mass testing, which may possibly account for its strikingly low number of confirmed cases. Its stringent infectious control has been backed up by the country's 
(a)

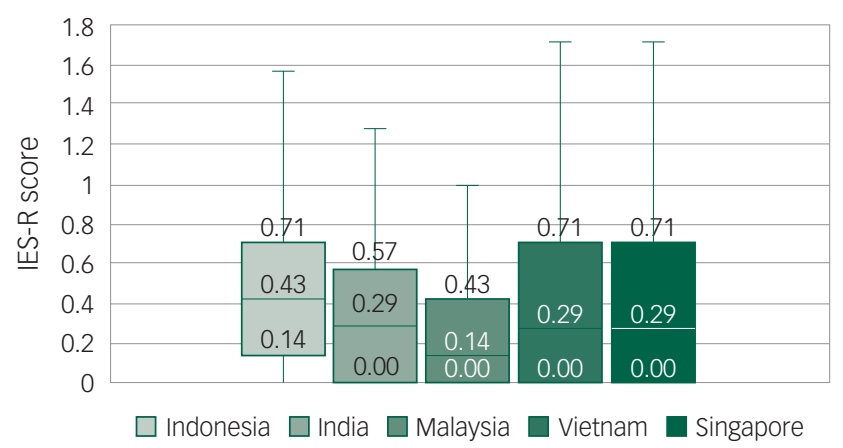

(b)

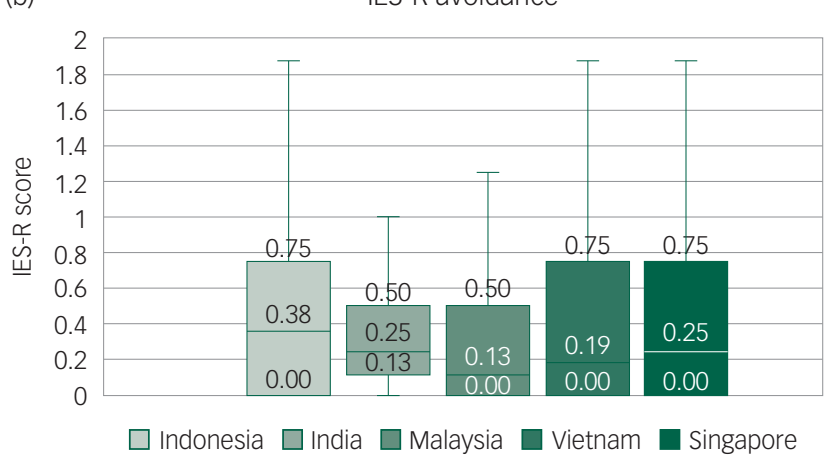

(c)

IES-R hyperarousal

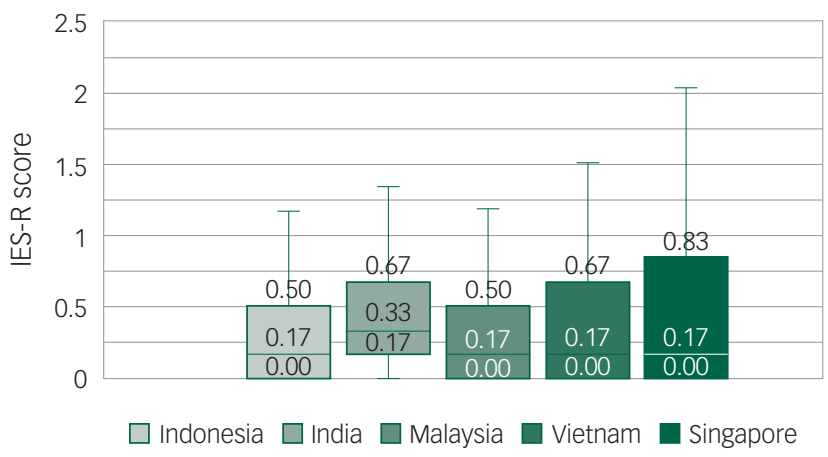

Fig. 5 The mean Impact of Events Scale Revised (ES-R) scores for each country, categorised into its subscales of (a) intrusion, (b) avoidance and (c) hyperarousal $(n=1146)$.

military and public security forces. ${ }^{17}$ Despite their low levels of detected cases, an interesting finding of our study is Vietnam's high prevalence of PTSD, and relative higher rates of anxiety and depression as compared with their India and Singapore counterparts.

Singapore adopted a multipronged aggressive surveillance strategy among various patient groups (for example all patients with pneumonia) that allowed clinician's discretion to order a test based on clinical suspicion, even if the case definition was not met. ${ }^{18}$ This strategy led to an increase in detected COVID-19 cases, many of which may not have been detected if the case definition was strictly followed. Although, it reported a high volume of confirmed cases, most were asymptomatic or mildly symptomatic. ${ }^{18}$ Perhaps, this accounted for the lower prevalence of anxiety, depression and PTSD related to COVID-19 among healthcare workers.

Similarly, Malaysia adopted an aggressive testing strategy, in which its tests per million capita far exceed its Association of South-East Asian Nations counterparts, resulting in higher reported confirmed cases but of milder severity and lower mortality. ${ }^{19}$ However, despite its lower rates of confirmed cases as compared with Singapore, we found a higher prevalence of depression, anxiety and stress among Malaysian healthcare workers.

Indonesia's declaration of a public health emergency on 21 March led to large-scale social distancing and movement restriction. ${ }^{20}$ Despite its higher death rates per 1 million population, the prevalence of depression and anxiety remain comparable with its counterparts. Therefore, there is no clear correlation between the volume of confirmed cases per day per million capita and the prevalence of adverse psychological outcomes. Perhaps the risk of psychological distress among healthcare workers goes beyond the country's COVID-19 disease burden, and may also be contributed to by other societal and cultural factors specific to different nations.

\section{Differences between medical and non-medical healthcare workers}

Our study demonstrated that non-medically trained healthcare workers were at higher risk of adverse psychological outcomes as compared with their medically trained counterparts. This is in agreement with our recent study on healthcare workers during the start of the pandemic in Singapore, as well as a recent study in China that demonstrated that front-line nurses had significantly decreased vicarious traumatisation scores in comparison with non-front-line nurses and the general public during the COVID-19 pandemic. ${ }^{5,21}$ This difference between medical and non-medical healthcare workers may be attributed to the relative lack of accessibility to first-hand medical information on the pandemic and less formal training and confidence in infectious control measures. ${ }^{5}$

\section{Role of physical symptoms}

Our current findings demonstrate that the presence of physical symptoms was an independent predictor of adverse psychological outcomes. This is in line with our previous study that reported this significant association during the earlier stages of the pandemic. ${ }^{22}$ We postulated a bidirectional association between physical symptoms and psychological stress, in which somatic symptoms may represent a way of communicating emotions. ${ }^{23}$ However, healthcare workers displaying physical symptoms may face a sense of fear, stigmatisation and ostracism from co-workers, which may exacerbate the psychological pain. ${ }^{24-26}$

\section{Role of prior medical conditions}

We found that healthcare workers with prior medical conditions were at risk of adverse psychological outcomes. Studies have 
Table 3 Multivariate analysis of healthcare workers in each country with depression during the CoVID-19 pandemic $(n=1146)$

\begin{tabular}{|c|c|c|c|c|c|c|c|c|c|c|c|c|}
\hline \multirow[b]{2}{*}{ Variables } & \multicolumn{2}{|c|}{ Overall $(n=1146)$} & \multicolumn{2}{|c|}{ Singapore $(n=277)$} & \multicolumn{2}{|l|}{ India $(n=384)$} & \multicolumn{2}{|c|}{ Malaysia $(n=175)$} & \multicolumn{2}{|l|}{ Vietnam $(n=60)$} & \multicolumn{2}{|c|}{ Indonesia $(n=250)$} \\
\hline & OR $(95 \% \mathrm{Cl})$ & $P$ & OR $(95 \% \mathrm{Cl})$ & $P$ & OR $(95 \% \mathrm{Cl})$ & $P$ & OR $(95 \% \mathrm{Cl})$ & $P$ & OR $(95 \% \mathrm{Cl})$ & $P$ & OR $(95 \% \mathrm{Cl})$ & $P$ \\
\hline Gender (female) & $0.755(0.515-1.105)$ & 0.148 & $0.221(0.027-1.769)$ & 0.155 & $0.452(0.027-7.699)$ & 0.583 & $2.347(0.912-6.038)$ & 0.077 & $0.349(0.011-11.470)$ & 0.555 & $0.365(0.039-3.387)$ & 0.376 \\
\hline Non-medically trained personnel & $1.832(1.267-2.653)$ & 0.001 & $1.186(0.339-4.153)$ & 0.790 & $1.188(0.087-16.234)$ & 0.897 & $0.272(0.033-2.271)$ & 0.229 & $0.662(0.309-1.415)$ & 0.287 & $5.399(0.569-51.271)$ & 0.142 \\
\hline Presence of prior medical conditions & $1.346(0.886-2.045)$ & 0.164 & $1.310(0.350-4.901)$ & 0.688 & $1.739(0.088-34.381)$ & 0.716 & $0.303(0.076-1.210)$ & 0.091 & $0.284(0.012-6.582)$ & 0.432 & $0.670(0.098-4.580)$ & 0.683 \\
\hline Presence of symptoms & $3.185(1.916-5.291)$ & $<0.001$ & $6.017(0.740-48.919)$ & 0.093 & $1.332(0.860-2.062)$ & 0.199 & $5.673(1.780-18.075)$ & 0.003 & $1.127(0.085-15.029)$ & 0.928 & $1.241(1.010-1.526)$ & 0.040 \\
\hline Age & $1.005(0.983-1.028)$ & 0.657 & $0.988(0.923-1.057)$ & 0.719 & $1.090(0.948-1.254)$ & 0.228 & $1.002(0.920-1.092)$ & 0.959 & $1.173(0.949-1.451)$ & 0.140 & $0.936(0.797-1.100)$ & 0.422 \\
\hline
\end{tabular}

Results in bold are significant.

Table 4 Multivariate analysis of healthcare workers in each country with anxiety during the COVID-19 pandemic $(n=1146)$

\begin{tabular}{|c|c|c|c|c|c|c|c|c|c|c|c|c|}
\hline \multirow[b]{2}{*}{ Variables } & \multicolumn{2}{|c|}{ Overall $(n=1146)$} & \multicolumn{2}{|c|}{ Singapore $(n=277)$} & \multicolumn{2}{|l|}{ India $(n=384)$} & \multicolumn{2}{|c|}{ Malaysia $(n=175)$} & \multicolumn{2}{|c|}{ Vietnam $(n=60)$} & \multicolumn{2}{|c|}{ Indonesia $(n=250)$} \\
\hline & OR $(95 \% \mathrm{Cl})$ & $P$ & OR $(95 \% \mathrm{Cl})$ & $P$ & OR $(95 \% \mathrm{Cl})$ & $P$ & OR $(95 \% \mathrm{Cl})$ & $P$ & OR $(95 \% \mathrm{Cl})$ & $P$ & OR $(95 \% \mathrm{Cl})$ & $P$ \\
\hline Gender (female) & $0.904(0.684-1.193)$ & 0.477 & $0.594(0.117-3.016)$ & 0.530 & $1.155(0.069-19.390)$ & 0.920 & $1.537(0.634-3.729)$ & 0.342 & $0.324(0.012-8.663)$ & 0.502 & $0.419(0.130-1.351)$ & 0.145 \\
\hline Non-medically trained personnel & $1.173(0.880-1.563)$ & 0.277 & $1.587(0.420-6.000)$ & 0.496 & $1.211(0.074-19.870)$ & 0.893 & $0.775(0.159-3.780)$ & 0.752 & $0.662(0.309-1.415)$ & 0.287 & $4.908(1.282-18.789)$ & 0.020 \\
\hline Presence of prior medical conditions & $1.126(0.814-1.558)$ & 0.474 & $5.828(1.397-24.308)$ & 0.016 & $5.774(0.430-77.551)$ & 0.186 & $1.432(0.483-4.249)$ & 0.517 & $0.259(0.009-7.576)$ & 0.433 & $0.906(0.304-2.693)$ & 0.858 \\
\hline Presence of symptoms & $1.408(1.052-1.890)$ & 0.022 & $4.502(0.544-37.257)$ & 0.163 & $0.693(0.046-10.503)$ & 0.791 & $1.735(0.685-4.393)$ & 0.245 & $0.808(0.050-12.946)$ & 0.880 & $4.660(0.587-37.014)$ & 0.145 \\
\hline Age & $0.996(0.978-1.013)$ & 0.627 & $1.001(0.938-1.069)$ & 0.966 & $0.917(0.693-1.214)$ & 0.546 & $0.903(0.816-1.000)$ & 0.051 & $1.120(0.941-1.332)$ & 0.201 & $0.999(0.931-1.071)$ & 0.967 \\
\hline
\end{tabular}




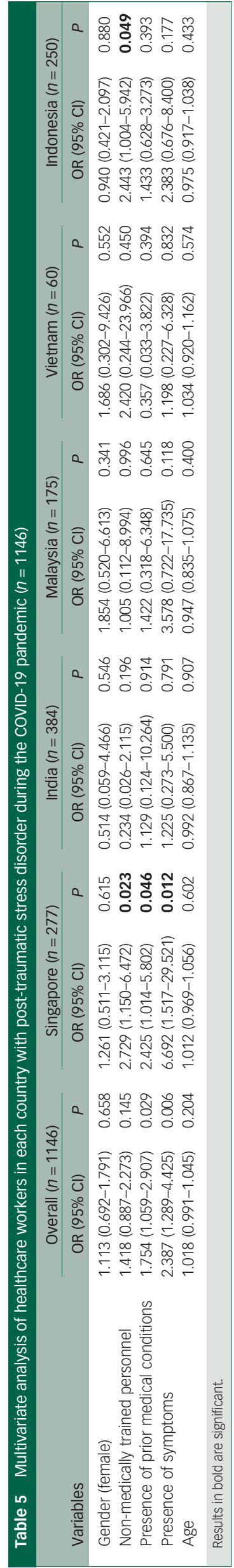

shown that vulnerable groups, namely those who are immunocompromised, with underlying medical conditions are at higher risk of life-threatening illness from COVID-19. ${ }^{27,28}$ The knowledge of this increased risk may have worked as an additional stressor for our healthcare workers with pre-existing illnesses. Perhaps more efforts can be made to address the concerns of this vulnerable group of healthcare workers through ensuring that their exposure to COVID-19 patients are adjusted accordingly to their health risk status.

\section{Implications}

Our present findings demonstrate that regardless of the volume of cases or deaths, healthcare workers from all countries are vulnerable to psychological distress from the COVID-19 outbreak. Therefore, as the pandemic reaches its peak, it calls for urgent clinical and policy strategies for identifying healthcare workers at risk, i.e. those who are not medically trained, those with physical symptoms and prior medical conditions. Passive psychoeducation through educational pamphlets, emails or website can be relatively easy and inexpensive to implement, and may serve as a readily available resource for those experiencing psychological distress. Education on the natural history of the virus, and the appropriate use of infection control measures, especially for the non-medically trained hospital workers, may be helpful. A meta-analysis has shown that brief passive psychoeducational interventions targeting high-risk groups can be effective, although the extent of benefit remains unknown. ${ }^{29}$ Early psychological interventions in the form of cognitive-behavioural therapy, have also been proven to reduce psychological distress. ${ }^{30}$ Therefore, improved accessibility to formal psychological support for healthcare workers (in the form of counselling, internet-based cognitive-behavioural therapy) is of paramount importance. ${ }^{5}$ These dedicated interventions will help allay the fear of COVID-19 transmission between colleagues and improve the confidence of our healthcare workers. ${ }^{12,22}$

\section{Limitations}

We acknowledge certain limitations of the study. First, it is crosssectional in nature, which does not allow us to assess the causality of the different psychological outcomes. We also did not examine the prevalence of psychological symptoms before COVID-19, and did not examine the participants longitudinally for progression or improvement in their symptoms. Second, questionnaires were self-administered because of the strict infection control measures in all participating institutions. Hence, the information obtained could not be verified by a medical professional. Furthermore, all surveys were administered in English. Although English had been the primary language of communication in all the hospitals in which the survey had been administered, varying levels of proficiency with the English language may also have contributed to bias and inaccuracy in the survey findings.

In terms of sampling, larger tertiary centres within the countries were studied. The findings thus may not be generalisable and reflective of smaller regional or rural centres. Although the response rate was greater than $88 \%$, participants with greater mental health concerns may be more likely to respond to the study, which may slightly overestimate the prevalence of mental health disorders. A larger sample size that is more representative of healthcare workers across the study region would be needed to estimate the true prevalence of mental disorders in this population. Follow-up studies are required to evaluate the progression of the psychological impact on healthcare workers during the aftermath of the COVID-19 pandemic, as there have been concerns regarding the 'rebound effect' where high-risk healthcare workers may experience various neuropsychiatric manifestations once the imminent threat of the infectious 
disease subsides, along with the protective positive attitude of collegiality and bravery. ${ }^{31}$

In conclusion, we reported a varied, albeit high, prevalence of psychological distress in healthcare workers regardless of the individual countries' burden of confirmed COVID-19 cases and deaths, as well as the different infectious control strategies adopted by the various countries. Our study suggested that vulnerable groups of healthcare workers may include those who are not medically trained, and those with physical symptoms and prior medical conditions. Targeted psychological interventions may be beneficial for this group of healthcare workers.

Nicholas W.S. Chew, MRCP, Department of Medicine, National University Health System, Singapore; Jinghao Nicholas Ngiam (D), MRCP, Department of Medicine, National University Health System, Singapore; Benjamin Yong-Qiang Tan, MRCP, Division of Neurology, Department of Medicine, National University Health System, Singapore; and Department of Medicine, Yong Loo Lin School of Medicine, National University of Singapore, Singapore; Sai-Meng Tham, MRCP, Department of Medicine, National University Health System, Singapore; Celine Yan-Shan Tan, MBBS, Department of Medicine, National University Health System, Singapore; Mingxue Jing MBBS, Division of Neurology, Department of Medicine, National University Health System, Singapore; Renarebecca Sagayanathan, BSc, Division of Neurology, Department of Medicine, National University Health System, Singapore; Jin Tao Chen, Division of Neurology, Department of Medicine, National University Health System, Singapore; Lily Y. H. Wong, BN, Division of Neurology, Department of Medicine, National University Health System, Singapore; Aftab Ahmad, MRCP, Department of Neurology, Ng Teng Fong General Hospital, Singapore; Faheem Ahmed Khan, FCEM, Department of Fong General Hospital, Singapore; Faheem Ahmed Khan, FCEM, Department of
Intensive Care Medicine, Ng Teng Fong General Hospital, Singapore; Maznah Marmin Intensive Care Medicine, Ng Teng Fong General Hospital, Singapore; Maznah Marm
BN, Department of Neurology, Ng Teng Fong General Hospital, Singapore; Fadhlina Binte Hassan, BN, Department of Neurology, Ng Teng Fong General Hospital, Singapore Tai Mei-Ling Sharon, MD, University of Malaya, Kuala Lumpur, Malaysia; Chin Han Lim MD, Tawau Hospital, Malaysia; Mohamad Iqbal Bin Mohaini, MD, Tawau Hospital, Malaysia; Rivan Danuaji, MD, Dr Moewardi Hospital Surakarta, Indonesia; Thang H. Nguyen, MD, Cerebrovascular Disease Department, 115 People's Hospital, Vietnam; Georgios Tsivgoulis, MD, Attikon University Hospital, National and Kapodistrian University of Athens, Greece; Sotirios Tsiodras, MD, Attikon University Hospital, National and Kapodistrian University of Athens, Greece; Paraskevi C. Fragkou (D), MD Attikon University Hospital, National and Kapodistrian University of Athens, Greece; Dimitra Dimopoulou, MD, Attikon University Hospital, National and Kapodistrian University of Athens, Greece; Arvind K. Sharma, MD, Zydus Hospital, India; Kenam Shah, MD, Zydus Hospital, India; Bhargesh Patel, MD, Zydus Hospital, India; Suktara Sharma, MD, GCS Medical College, India; R. N. Komalkumar, MD, Yashoda Hospital, India; R. V. Meenakshi, MD, Senthil Multi Specialty Hospital, India; Shikha Talati, MD, Department of Psychiatry, Geetanjali Medical College and Hospital India; Hock Luen Teoh, MD, Division of Neurology, Department of Medicine, National University Health System, Singapore; and Department of Medicine, Yong Loo Lin Schoo of Medicine, National University of Singapore, Singapore; Cyrus S. Ho, MRCPsych, Department of Psychological Medicine, National University of Singapore, Singapore; Roger C. Ho, FRCPsych, Department of Psychological Medicine and Institute of Health Innovation and Technology (iHealthtech), National University of Singapore, Singapore; Vijay K. Sharma, MD, Division of Neurology, Department of Medicine, National University Health System, Singapore; and Department of Medicine, Yong Loo Lin School of Medicine, National University of Singapore, Singapore

Correspondence: Benjamin Y. Q. Tan. Email: benjamin_yq_tan@nuhs.edu.sg

First received 15 Jul 2020, final revision 22 Aug 2020, accepted 24 Aug 2020

\section{Data availability}

The data that support the findings of this study are available on request from the corresponding author, B.Y.Q.T.

\section{Author contributions} N.W.S.C., J.N.N., B.Y.Q.T. were involved in the conception of the study, data collection, analysis
and writing of the manuscript. S.M.T. C.Y.S.T.M.J.R.S. J.T.C. L.Y.H.W.,A.A.F.A.K.,M.M.,F.B. and writing of the manuscript. S.-M.T., C.Y.-S.T., M.J., R.S., J.T.C., L.Y.H.W., A.A., F.A.K., M.M., F.B.
H., T.M.-L.S., C.H.L., M.I.B.M., R.D. and T.H.N., were involved in conception, data collection, analysis and critical revision of the manuscript. G.T., S.T., P.C.F. and D.D. were involved the conception of the study, writing and critical revision of the manuscript. A.K.S., K.S., B.P., S.S., R.N.K., R.V. M., S.T., H.L.T., C.S.H., R.C.H. and V.K.S. were involved in the conception of the study, data collection, analysis, writing and critical revision of the manuscript.

\section{Funding}

There was no funding for this study. All authors have no financial disclosures to declare.

\section{Declaration of interest}

V.K.S. is the current recipient of a Senior Clinician Scientist Award from the National Medical Research Council, Ministry of Health, Singapore. All authors have no other conflicts of interests to declare.

\section{References}

1 World Health Organization. Director-General's Remarks at the Media Briefing on 2019-nCoV on 11 February 2020. WHO, 2020 (https://www.who.int/dg/ speeches/detail/who-director-general-s-remarks-at-the-media-briefing-on2019-ncov-on-11-february-2020).

2 Worldometer. COVID-19 Coronavirus Pandemic. Worldometer, 2020 (https:// www.worldometers.info/coronavirus/ [cited 16 June 16 2020])

3 Driggin E, Madhavan MV, Bikdeli B, Chuich T, Laracy J, Biondi-Zoccai G, et al. Cardiovascular considerations for patients, health care workers, and health systems during the coronavirus disease 2019 (COVID-19) pandemic. J Am Coll Cardiol 2020; 75: 2352-71.

4 Zeng J, Huang J, Pan L. How to balance acute myocardial infarction and COVID19: the protocols from Sichuan Provincial People's Hospital. Intensive Care Med 2020; 46: 1111-3.

5 Tan BYQ, Chew NWS, Lee GKH, Jing M, Goh Y, Yeo LLL, et al. Psychological impact of the COVID-19 pandemic on health care workers in Singapore. Ann Intern Med 2020; 173: 317-20.

6 Grace SL, Hershenfield K, Robertson E, Stewart DE. The occupational and psychosocial impact of SARS on academic physicians in three affected hospitals. Psychosomatics 2005; 46: 385-91.

7 Tam CW, Pang EP, Lam LC, Chiu HF. Severe acute respiratory syndrome (SARS) in Hong Kong in 2003: stress and psychological impact among frontline healthcare workers. Psychol Med 2004; 34: 1197-204.

8 Lam MHB, Wing YK, Yu MWM, Leung CM, Ma RCW, Kong APS, et al. Mental morbidities and chronic fatigue in severe acute respiratory syndrome survivors: long-term follow-up. Archiv Int Med 2009; 169: 2142-7.

9 Lee S, Chan LY, Chau AM, Kwok KP, Kleinman A. The experience of SARSrelated stigma at Amoy Gardens. Soc Sci Med 2005; 61: 2038-46.

10 Lovibond SH, Lovibond PF. Manual for the Depression Anxiety Stress Scales (2nd edn). Psychology Foundation of Australia, 1995.

11 Creamer M, Bell R, Failla S. Psychometric properties of the Impact of Event Scale - Revised. Behav Res Ther 2003; 41: 1489-96.

12 Wang C, Pan R, Wan X, Tan $Y, X u$ L, Ho CS, et al. Immediate psychological responses and associated factors during the initial stage of the 2019 coronavirus disease (COVID-19) epidemic among the general population in China. Int J Environ Res Public Health 2020; 17: 1729.

13 Young BE, Ong SWX, Kalimuddin S, Low JG, Tan SY, Loh J, et al. Epidemiologic features and clinical course of patients infected with SARS-CoV-2 in Singapore. JAMA 2020; 323: 1488-94.

14 Wong JEL, Leo YS, Tan CC. COVID-19 in Singapore-current experience: critica global issues that require attention and action. JAMA [Epub ahead of print] 20 Feb 2020. Available from: https://doi.org/10.1001/jama.2020.3204.

15 Huang JZ, Han MF, Luo TD, Ren AK, Zhou XP. Mental health survey of 230 medical staff in a tertiary infectious disease hospital for COVID-19 [in Chinese]. Zhonghua Lao Dong Wei Sheng Zhi Ye Bing Za Zhi 2020; 38: E001.

16 Lancet T. India under COVID-19 lockdown. Lancet 2020; 395: 1315.

17 Black G. Vietnam may have the most effective response to Covid-19. The Nation 2020; 24 April 2020 (https://www.thenation.com/article/world/coronavirusvietnam-quarantine-mobilization).

$18 \mathrm{Ng} Y, \mathrm{Li}$ Z, Chua YX, Chaw WL, Zhao Z, Er B, et al. Evaluation of the effectiveness of surveillance and containment measures for the first 100 patients with COVID-19 in Singapore - January 2-February 29, 2020. MMWR Morb Mortal Wkly Rep 2020; 69: 207-11.

19 New Straits Times. Malaysia's Covid-19 strategy far exceeds ASEAN, some European peers. New Straits Times, 25 March 2020 (https://www.nst.com.my/ business/2020/03/577996/jp-morgan-malaysias-covid-19-strategy-far-exceedsasean-some-european-peers).

20 Sutrisno B. Indonesia's strategy to combat COVID-19: what we know so far. The Jarkata Post 2020: 3 April 2020 (https://www thejakartapost com/news/2020/ 04/03/indonesias-strategy-to-combat-covid-19-what-we-know-so-far.html).

21 Li Z, Ge J, Yang M, Feng J, Qiao M, Jiang R, et al. Vicarious traumatization in the general public, members, and non-members of medical teams aiding in COVID19 control. Brain Behav Immun 2020; 88: 916-9.

22 Chew NWS, Lee GKH, Tan BYQ, Jing M, Goh Y, Ngiam NJH, et al. A multinational, multicentre study on the psychological outcomes and associated physical symptoms amongst healthcare workers during COVID-19 outbreak. Brain Behav Immun 2020; 88: 559-65.

23 Puri BK, Hall A, Ho R. Revision Notes in Psychiatry. Taylor \& Francis, 2013.

24 Nezlek JB, Wesselmann ED, Wheeler L, Williams KD. Ostracism in everyday life: the effects of ostracism on those who ostracize. J Soc Psychol 2015; 155: 432-51.

25 Troyer EA, Kohn JN, Hong S. Are we facing a crashing wave of neuropsychiatric sequelae of COVID-19? Neuropsychiatric symptoms and potential immunologic 
mechanisms. Brain Behav Immun 2020; 87: 34-9.

26 Matua GA, Van Der Wal DM. Living under the constant threat of Ebola: a phenomenological study of survivors and family caregivers during an ebola outbreak. J Nurs Res 2015; 23: 217-24.

27 Zhang J, Lu H, Zeng H, Zhang S, Du Q, Jiang T, et al. The differential psychological distress of populations affected by the COVID-19 pandemic. Brain Behav Immun 2020; 87: 49-50.

28 Richards $\mathrm{M}$, Anderson $\mathrm{M}$, Carter $\mathrm{P}$, Ebert BL, Mossialos $\mathrm{E}$. The impact of the COVID-19 pandemic on cancer care. Nat Cancer 2020; 1: 565-7.
29 Donker T, Griffiths KM, Cuijpers P, Christensen H. Psychoeducation for depression, anxiety and psychological distress: a meta-analysis. BMC Med 2009; 7: 79.

30 Mcnally RJ, Bryant RA, Ehlers A. Does early psychological intervention promote recovery from posttraumatic stress? Psychol Sci Public Interes 2003; 4: 45-79.

31 Maunder RG, Lancee WJ, Balderson KE, Bennett JP, Borgundvaag B, Evans S, et al. Long-term psychological and occupational effects of providing hospital healthcare during SARS outbreak. Emerg Infect Dis 2006; 12: 1924-32. 